\title{
Second-order neutral impulsive stochastic evolution equations with infinite delay
}

\section{Chaohui Yue*}

*Correspondence:

huihui.eyre5166@163.com

School of Sciences, Anhui

Agricultural University, Hefei,

230036, China

\section{Springer}

\begin{abstract}
In this paper, we study a class of second-order neutral impulsive stochastic evolution equations with infinite delay (SNISEEls in short), in which the initial value belongs to the abstract space $\mathcal{B}_{h}$. Sufficient conditions for the existence of the mild solutions for SNISEEls are derived by means of the Krasnoselskii-Schaefer fixed point theorem. Two examples are given to illustrate the obtained results.
\end{abstract}

Keywords: second-order stochastic evolution equation; impulsive effect; cosine family; fixed point theorem

\section{Introduction}

In this paper, we consider the second-order neutral impulsive stochastic evolution equations with infinite delay (SNISEEIs in short) of the following form:

$$
\begin{aligned}
& \mathrm{d}\left[x^{\prime}(t)-g\left(t, x_{t}\right)\right]=\left[A x(t)+f\left(t, x_{t}\right)\right] \mathrm{d} t+\sigma\left(t, x_{t}\right) \mathrm{d} w(t), \quad t \in J:=[0, T], \\
& x_{0}=\phi \in \mathcal{B}_{h}, \\
& x^{\prime}(0)=\psi \in H, \\
& \triangle x\left(t_{k}\right)=I_{k}\left(x_{t_{k}}\right), \quad \triangle x^{\prime}\left(t_{k}\right)=\tilde{I}_{k}\left(x_{t_{k}}\right), \quad k=1,2, \ldots, m .
\end{aligned}
$$

Here, the state $x(\cdot)$ takes values in a separable real Hilbert space $H$ with inner product $(\cdot, \cdot)$ and norm $\|\cdot\|$, where $A: D(A) \subset H \rightarrow H$ is the infinitesimal generator of a strongly continuous cosine family $C(t)$ on $H$. The history $x_{t}:(-\infty, 0] \rightarrow H, x_{t}(\theta)=x(t+\theta)$, for $t \geq 0$, belongs to the phase space $\mathcal{B}_{h}$. Now, we present the abstract phase space $\mathcal{B}_{h}$. Assume that $h:(-\infty, 0] \rightarrow(0, \infty)$ is a continuous function with $l=\int_{-\infty}^{0} h(t) \mathrm{d} t<\infty$. For any $a>0$, define

$$
\begin{gathered}
\mathcal{B}_{h}=\left\{\psi:(-\infty, 0] \rightarrow H:\left(E\|\psi(\theta)\|^{2}\right)^{1 / 2}\right. \text { is a bounded and measurable } \\
\text { function on } \left.[-a, 0] \text { and } \int_{-\infty}^{0} h(s) \sup _{s \leq \theta \leq 0}\left(E\|\psi(\theta)\|^{2}\right)^{1 / 2} \mathrm{~d} s<\infty\right\} .
\end{gathered}
$$

We endow $\mathcal{B}_{h}$ with the norm

$$
\|\psi\|_{\mathcal{B}_{h}}=\int_{-\infty}^{0} h(s) \sup _{s \leq \theta \leq 0}\left(E\|\psi(\theta)\|^{2}\right)^{1 / 2} \mathrm{~d} s, \quad \text { for all } \psi \in \mathcal{B}_{h}
$$

C2014 Yue; licensee Springer. This is an Open Access article distributed under the terms of the Creative Commons Attribution License (http://creativecommons.org/licenses/by/2.0), which permits unrestricted use, distribution, and reproduction in any medium, provided the original work is properly cited. 
then $\left(\mathcal{B}_{h},\|\cdot\|_{\mathcal{B}_{h}}\right)$ is a Banach space [1]. Let $K$ be another separable Hilbert space with inner product $(\cdot, \cdot)_{K}$ and norm $\|\cdot\|_{K}$. Suppose $\{w(t): t \geq 0\}$ is a given $K$-valued Wiener process with a finite trace nuclear covariance operator $Q \geq 0$ defined on a complete probability space $(\Omega, \mathcal{F}, P)$ equipped with a normal filtration $\left\{\mathcal{F}_{t}\right\}_{t \geq 0}$, which is generated by the Wiener process $w$. We are also employing the same notation $\|\cdot\|$ for the operator norm $L(K ; H)$, where $L(K ; H)$ denotes the space of all bounded linear operators from $K$ into $H$. Assume that $g, f: J \times \mathcal{B}_{h} \rightarrow H(i=1,2)$ and $\sigma: J \times \mathcal{B}_{h} \rightarrow L_{Q}(K, H)$ are appropriate mappings specified later. Here, $L_{Q}(K, H)$ denotes the space of all Q-Hilbert-Schmidt operators from $K$ into $H$, which will be defined in the next section. The initial data $\phi=\{\phi(t):-\infty<t \leq 0\}$ is an $\mathcal{F}_{0}$-adapted, $\mathcal{B}_{h}$-valued stochastic process independent of the Wiener process $w$ with finite second moment. $\psi$ is an $\mathcal{F}_{0}$-adapted, $H$-valued random variable independent of the Wiener process $w$ with finite second moment. $I_{k}$ and $\tilde{I}_{k}: \mathcal{B}_{h} \rightarrow H$ are appropriate functions. Moreover, let $0=t_{0}<t_{1}<\cdots<t_{m}<t_{m+1}=T$, be given time points and the symbol $\Delta \xi(t)$ represents the jump of the function $\xi$ at $t$, which is defined by $\Delta \xi(t)=\xi\left(t^{+}\right)-\xi\left(t^{-}\right)$.

Stochastic partial differential equations (SPDEs in short) with delay have attracted great interest due to their applications in describing many sophisticated dynamical systems in physical, biological, medical and social sciences. One can see [2-5] and the references therein for details. Moreover, to describe the systems involving derivatives with delay, Hale and Lunel [6] introduced the deterministic neutral functional differential equations, which are of great interest in theoretical and practical applications. Taking the environmental disturbances into account, Kolmanovskii and Myshkis [7] introduced the neutral stochastic functional differential equations (NSFDEs in short) and gave its applications in chemical engineering and aero elasticity. The investigation of qualitative properties such as existence, uniqueness and stability for NSFDEs has received much attention. One can see $[2,5,8-12]$ and the references therein. In addition, impulsive effects exist in many evolution processes in which states are changed abruptly at certain moments of time, involved in such fields as medicine and biology, economics, bioengineering, chemical technology etc. (see $[13,14]$ and the references therein).

On the other hand, the study of abstract deterministic second-order evolutions equations governed by the generator of a strongly continuous cosine family was initiated by [15] and subsequently studied by [16, 17]. The second-order stochastic differential equations are the right model in continuous time to account for integrated processes that can be made stationary. For instance, it is useful for engineers to model mechanical vibrations or charge on a capacitor or condenser subjected to white noise excitation through a second-order stochastic differential equations. There are some interesting works that have been done on the second-order stochastic differential equations. For example, McKibben [18] investigated the second-order damped functional stochastic evolution equations. For further work on this topic, one can see Mahmudov and McKibben [19]. Moreover, McKibben [20] established the existence and uniqueness of mild solutions for a class of second-order neutral stochastic evolution equations with finite delay. Balasubramaniam and Muthukumar [21] gave the sufficient conditions for the approximate controllability of the second-order neutral stochastic evolution equations with infinite delay. For more details of second-order stochastic differential equations, we refer the reader to Da Prato [22] and the references therein.

To the best of our knowledge, there is no work reported in the literature about SNISEEIs and the aim of this paper is to close this gap. We aim to establish the existence of the mild 
solutions for SNISEEIs by means of the Krasnoselskii-Schaefer fixed point theorem. Two types of stochastic nonlinear wave equations with infinite delay and impulsive effects are provided to illustrate the obtained results.

The paper is organized as follows. In Section 2, we introduce some preliminaries. In Section 3, we prove the existence of the mild solutions for SNISEEIs by means of the Krasnoselskii-Schaefer fixed point theorem. In Section 4, we study the continuous dependence of solutions on the initial values. Two examples are provided in the last section to illustrate the theory.

\section{Preliminaries}

In this section, we mention some preliminaries needed to establish our results. For details as regards this section, the reader may refer to Da Prato and Zabczyk [3], Fattorini [16] and the references therein.

Let $(\Omega, \mathcal{F}, P ; \mathbf{F})\left(\mathbf{F}=\left\{\mathcal{F}_{t}\right\}_{t \geq 0}\right)$ be a complete filtered probability space satisfying that $\mathcal{F}_{0}$ contains all $P$-null sets of $\mathcal{F}$. An $H$-valued random variable is an $\mathcal{F}$-measurable function $x(t): \Omega \rightarrow H$ and the collection of random variables $S=\{x(t, \omega): \Omega \rightarrow H \mid t \in J\}$ is called a stochastic process. Generally, we just write $x(t)$ instead of $x(t, \omega)$ and $x(t): J \rightarrow H$ in the space of $S$. Let $\left\{e_{i}\right\}_{i=1}^{\infty}$ be a complete orthonormal basis of $K$. Suppose that $\{w(t): t \geq 0\}$ is a cylindrical $K$-valued Wiener process with a finite trace nuclear covariance operator $Q \geq 0$, denote $\operatorname{Tr}(Q)=\sum_{i=1}^{\infty} \lambda_{i}=\lambda<\infty$, which satisfies $Q e_{i}=\lambda_{i} e_{i}$, with $e_{i}$ being a CONS of eigenvectors, and then, w.r.t. this spectral representation of $Q$ the driving $Q$-Wiener process can be represented as $w(t)=\sum_{i=1}^{\infty} \sqrt{\lambda_{i}} w_{i}(t) e_{i}$, where $\left\{w_{i}(t)\right\}_{i=1}^{\infty}$ are mutually independent onedimensional standard Wiener processes. We assume that $\mathcal{F}_{t}=\sigma\{w(s): 0 \leq s \leq t\}$, which is a $\sigma$-algebra generated by $w$ and $\mathcal{F}_{T}=\mathcal{F}$. Let $\psi \in L(K, H)$ and define

$$
\|\psi\|_{Q}^{2}=\operatorname{Tr}\left(\psi Q \psi^{*}\right)=\sum_{n=1}^{\infty}\left\|\sqrt{\lambda_{n}} \psi e_{n}\right\|^{2} .
$$

If $\|\psi\|_{Q}<\infty$, then $\psi$ is called a $Q$-Hilbert-Schmidt operator. Let $L_{Q}(K, H)$ denote the space of all Q-Hilbert-Schmidt operators $\psi: K \rightarrow H$. The completion $L_{Q}(K, H)$ of $L(K, H)$ with respect to the topology induced by the norm $\|\cdot\|_{Q}$ with $\|\psi\|_{Q}^{2}=(\psi, \psi)$ is a Hilbert space with the above norm topology.

The collection of all strongly measurable, square-integrable, $H$-valued random variables, denoted by $L_{2}(\Omega, \mathcal{F}, P ; H) \equiv L_{2}(\Omega, H)$, is a Banach space equipped with norm $\|x(\cdot)\|_{L_{2}}=\left(E\|x(\cdot, \omega)\|_{H}^{2}\right)^{1 / 2}$, where the expectation $E$ is defined by $E x=\int_{\Omega} x(\omega) \mathrm{d} P$. Let $C\left(J, L_{2}(\Omega, H)\right)$ be the Banach space of all continuous maps from $J$ into $L_{2}(\Omega, H)$ satisfying the condition $\sup _{t \in J} E\|x(t)\|^{2}<\infty$. An important subspace is given by $L_{2}^{0}(\Omega, H)=\{f \in$ $L_{2}(\Omega, H): f$ is $\mathcal{F}_{0}$-measurable\}.

We say that a function $x:[\nu, \tau] \rightarrow H$ is a normalized piecewise continuous function on $[\nu, \tau]$ if $x$ is piecewise continuous and left continuous on $(\nu, \tau]$. We denote by $\mathcal{P C}([\nu, \tau] ; H)$ the space formed by the normalized piecewise continuous stochastic processes from $\{x(t)$ : $t \in[v, \tau]\}$. In particular, we introduce the space $\mathcal{P C}$ formed by all $H$-valued stochastic processes $\{x(t): t \in[0, T]\}$ such that $x$ is continuous at $t \neq t_{k}, x\left(t_{k}^{-}\right)=x\left(t_{k}\right)$ and $x\left(t_{k}^{+}\right)$exists, for all $k=1, \ldots, m$. In the sequel, we always assume that $\mathcal{P C}$ is endowed with the norm $\|x\|_{\mathcal{P C}}=\left(\sup _{s \in J} E\|x(s)\|^{2}\right)^{1 / 2}$. It is clear that $\left(\mathcal{P C},\|\cdot\|_{\mathcal{P C}}\right)$ is a Banach space. 
To simplify the notations, we put $t_{0}=0, t_{n+1}=T$. For $x \in \mathcal{P C}$, we denote $\tilde{x}_{k} \in$ $C\left(\left[t_{k}, t_{k+1}\right] ; L_{2}(\Omega, H)\right), k=0,1, \ldots, n$, given by

$$
\tilde{x}_{k}(t)= \begin{cases}x(t), & \text { for } t \in\left(t_{k}, t_{k+1}\right], \\ x\left(t_{k}^{+}\right), & \text {for } t=t_{k} .\end{cases}
$$

Moreover, for $B \subseteq \mathcal{P C}$, we denote by $\widetilde{B}_{k}, k=0,1, \ldots, n$, the set $\widetilde{B}_{k}=\left\{\tilde{x}_{k}: x \in B\right\}$.

Lemma $1 A$ set $B \subseteq \mathcal{P C}$ is relatively compact in $\mathcal{P C}$, if and only if, the set $\widetilde{B}_{k}$ is relatively compact in $C\left(\left[t_{k}, t_{k+1}\right] ; L_{2}(\Omega, H)\right)$, for every $k=0,1, \ldots, m$.

Now, we consider the space

$$
\begin{aligned}
\mathcal{B}_{b}= & \left\{x:(-\infty, T] \rightarrow H, x_{k} \in \mathcal{P C}\left(J_{k}, H\right) \text { and there exist } x\left(t_{k}^{-}\right) \text {and } x\left(t_{k}^{+}\right)\right. \\
& \text {with } \left.x\left(t_{k}\right)=x\left(t_{k}^{-}\right), x_{0}=\varphi \in \mathcal{B}_{h}, k=0,1,2, \ldots, m\right\},
\end{aligned}
$$

where $x_{k}$ is the restriction of $x$ to $J_{k}=\left(t_{k}, t_{k+1}\right]$. Set $\|\cdot\|_{b}$ be a semi-norm in $\mathcal{B}_{b}$ defined by

$$
\|x\|_{b}=\left\|x_{0}\right\|_{\mathcal{B}_{h}}+\sup _{0 \leq s \leq T}\left(E\|x(s)\|^{2}\right)^{1 / 2}, \quad x \in \mathcal{B}_{b}
$$

Then we have the following useful lemma appearing in [23].

Lemma 2 Assume that $x \in \mathcal{B}_{b}$, then for $t \in J, x_{t} \in \mathcal{B}_{h}$. Moreover, we have

$$
l\left(E\|x(t)\|^{2}\right)^{1 / 2} \leq\left\|x_{t}\right\|_{\mathcal{B}_{h}} \leq l \sup _{0 \leq s \leq t}\left(E\|x(s)\|^{2}\right)^{1 / 2}+\left\|x_{0}\right\|_{\mathcal{B}_{h}}
$$

where $l=\int_{-\infty}^{0} h(s) \mathrm{d} s<\infty$

Now, let us recall some facts about cosine families of operators $C(t)$ and $S(t)$ appeared in $[15,16]$.

Definition 3 A one parameter family $\{C(t): t \in \mathbb{R}\} \subset L(H, H)$ satisfying that

(i) $C(0)=I$,

(ii) $C(t) x$ is continuous in $t$ on $\mathbb{R}$, for all $x \in H$,

(iii) $C(t+s)+C(t-s)=2 C(t) C(s)$, for all $t, s \in \mathbb{R}$,

is called a strongly continuous cosine family.

The corresponding strongly continuous sine family $\{S(t): t \in \mathbb{R}\} \subset L(H, H)$ is defined by $S(t) x=\int_{0}^{t} C(s) x \mathrm{~d} s, t \in \mathbb{R}, x \in H$.

The generator $A: H \rightarrow H$ of $\{C(t): t \in \mathbb{R}\}$ is given by $A x=\left.\frac{\mathrm{d}^{2}}{\mathrm{~d} t^{2}} C(t) x\right|_{t=0}$ for all $x \in D(A)=$ $\left\{x \in H: C(\cdot) x \in C^{2}(\mathbb{R} ; H)\right\}$.

It is well known that the infinitesimal generator $A$ is a closed, densely defined operator on $H$. Such cosine and corresponding sine families and their generators satisfy the following properties appearing in Fattorini [16]:

Proposition 4 Suppose that $A$ is the infinitesimal generator of a cosine family of operators $\{C(t): t \in \mathbb{R}\}$. Then we have 
(i) there exist $M^{*} \geq 1$ and $\alpha \geq 0$ such that $\|C(t)\| \leq M^{*} \mathrm{e}^{\alpha|t|}$ and hence $\|S(t)\| \leq M^{*} \mathrm{e}^{\alpha|t|}$,

(ii) $A \int_{s}^{\hat{r}} S(u) x \mathrm{~d} u=[C(\hat{r})-C(s)] x$ for all $0 \leq s \leq \hat{r}<\infty$,

(iii) there exists $N^{*} \geq 1$ such that $\|S(s)-S(\hat{r})\| \leq N^{*} \int_{s}^{\hat{r}} \mathrm{e}^{\alpha|s|} \mathrm{d} s$, for all $0 \leq s \leq \hat{r}<\infty$.

The uniform boundedness principle, together with Proposition 4(i), implies that both $\{C(t): t \in[0, T]\}$ and $\{S(t): t \in[0, T]\}$ are uniformly bounded.

To prove our results, we need the following Krasnoselskii-Schaefer type fixed point theorem appearing in [24].

Theorem 5 Let $\Phi_{1}$ and $\Phi_{2}$ be two operators of $H$ such that

(i) $\Phi_{1}$ is a strict contraction, and

(ii) $\Phi_{2}$ is completely continuous.

Then either

(1) the operator equation $\Phi_{1} x+\Phi_{2} x=x$ has a solution, or

(2) the set $G=\left\{x \in H: \lambda \Phi_{1}\left(\frac{x}{\lambda}\right)+\lambda \Phi_{2} x=x\right\}$ is unbounded for $\lambda \in(0,1)$.

\section{Existence result}

In this section, we aim to give the existence of mild solutions for SNISEEIs (1)-(4). Firstly, let us propose the definition of the mild solution of SNISEEIs (1)-(4).

Definition 6 An $\mathcal{F}_{t}$-adapted stochastic process $x:(-\infty, T] \rightarrow H$ is called a mild solution of SNISEEIs (1)-(4) if

(i) $\left\{x_{t}: t \in J\right\}$ is $\mathcal{B}_{h}$-valued and $\left.x(\cdot)\right|_{J} \in \mathcal{P C}$;

(ii) $x(t) \in H$ has càdlàg paths on $t \in J$ a.s. and for each $t \in J, x(t)$ satisfies the following integral equation:

$$
\begin{aligned}
x(t)= & C(t) \phi(0)+S(t)[\psi-g(0, \phi)]+\int_{0}^{t} C(t-s) g\left(s, x_{s}\right) \mathrm{d} s \\
& +\int_{0}^{t} S(t-s) f\left(s, x_{s}\right) \mathrm{d} s+\int_{0}^{t} S(t-s) \sigma\left(s, x_{s}\right) \mathrm{d} w(s) \\
& +\sum_{0<t_{k}<t} C\left(t-t_{k}\right) I_{k}\left(x_{t_{k}}\right)+\sum_{0<t_{k}<t} S\left(t-t_{k}\right) \tilde{I}_{k}\left(x_{t_{k}}\right) ;
\end{aligned}
$$

(iii) $x_{0}=\phi, x^{\prime}(0)=\psi$.

In this paper, we need the following assumptions:

(H1) The cosine family of operators $\{C(t): t \in[0, T]\}$ on $H$ and the corresponding sine family $\{S(t): t \in[0, T]\}$ satisfy $\|C(t)\|^{2} \leq M,\|S(t)\|^{2} \leq M, t \geq 0$ for a positive constant $M$.

(H2) The function $f: J \times \mathcal{B}_{h} \rightarrow H$ satisfies the following properties:

1. $f(\cdot, \phi): J \rightarrow H$ is strongly measurable for every $\phi \in \mathcal{B}_{h}$;

2. $f(t, \cdot): \mathcal{B}_{h} \rightarrow H$ is continuous for each $t \in J$;

3. there exist an integrable function $m: J \rightarrow[0, \infty)$ and a continuous nondecreasing function $\Psi:[0, \infty) \rightarrow(0, \infty)$ such that for every $(t, \phi) \in J \times \mathcal{B}_{h}$, we have

$$
E\|f(t, \phi)\|^{2} \leq m(t) \Psi\left(\|\phi\|_{\mathcal{B}_{h}}^{2}\right), \quad \liminf _{\zeta \rightarrow \infty} \frac{\Psi(\zeta)}{\zeta}=\Lambda<\infty .
$$


(H3) The function $\sigma: J \times \mathcal{B}_{h} \rightarrow L_{Q}(K, H)$ satisfies the following properties:

1. $\sigma(t, \cdot): \mathcal{B}_{h} \rightarrow L_{Q}(K, H)$ is continuous for almost all $t \in J$;

2. $\sigma(\cdot, x): J \rightarrow L_{Q}(K, H)$ is strongly $\mathcal{F}_{t}$-measurable for each $x \in \mathcal{B}_{h}$;

3. there exists a positive constant $L_{\sigma}$ such that

$$
\begin{aligned}
& E\left\|\sigma\left(t, x_{1}\right)-\sigma\left(t, x_{2}\right)\right\|^{2} \leq L_{\sigma}\left\|x_{1}-x_{2}\right\|_{\mathcal{B}_{h}}^{2}, \quad\left(t, x_{i}\right) \in J \times \mathcal{B}_{h}, i=1,2 \\
& E\|\sigma(t, x)\|^{2} \leq L_{\sigma}\left(\|x\|_{\mathcal{B}_{h}}^{2}+1\right), \quad(t, x) \in J \times \mathcal{B}_{h} .
\end{aligned}
$$

(H4) The function $g: J \times \mathcal{B}_{h} \rightarrow H$ is continuous and there exists a positive constant $L_{g}$ such that

$$
\begin{aligned}
& E\left\|g\left(t, x_{1}\right)-g\left(t, x_{2}\right)\right\|^{2} \leq L_{g}\left\|x_{1}-x_{2}\right\|_{\mathcal{B}_{h}}^{2}, \quad\left(t, x_{i}\right) \in J \times \mathcal{B}_{h}, i=1,2 ; \\
& E\|g(t, x)\|^{2} \leq L_{g}\left(\|x\|_{\mathcal{B}_{h}}^{2}+1\right), \quad(t, x) \in J \times \mathcal{B}_{h} .
\end{aligned}
$$

(H5) The functions $I_{k}$ and $\tilde{I}_{k}: \mathcal{B}_{h} \rightarrow H$ are continuous and there are positive constants $L_{I_{k}}, L_{\tilde{I}_{k}}, k=1,2, \ldots, m$ such that

$$
\begin{aligned}
& E\left\|I_{k}(x)-I_{k}(y)\right\|^{2} \leq L_{I_{k}}\|x-y\|_{\mathcal{B}_{h}}^{2}, \quad x, y \in \mathcal{B}_{h}, k=1,2, \ldots, m, \\
& E\left\|\tilde{I}_{k}(x)-\tilde{I}_{k}(y)\right\|^{2} \leq L_{\tilde{I}_{k}}\|x-y\|_{\mathcal{B}_{h}}^{2}, \quad x, y \in \mathcal{B}_{h}, k=1,2, \ldots, m .
\end{aligned}
$$

The main result of this section is the following theorem.

Theorem 7 Assume the conditions (H1)-(H5) hold and assume that $S(t)$ is compact. Then there exists a mild solution of SNISEEIs (1)-(4) provided that

$$
12 M l^{2}\left[T \Lambda \int_{0}^{T} m(s) \mathrm{d} s+2 \sum_{k=1}^{m}\left(L_{I_{k}}+L_{\tilde{I}_{k}}\right)+2 T\left(T L_{g}+\operatorname{Tr}(Q) L_{\sigma}\right)\right]<1
$$

and

$$
L_{0}=8 M l^{2}\left[T\left(T L_{g}+\operatorname{Tr}(Q) L_{\sigma}\right)+\sum_{k=1}^{m}\left(L_{I_{k}}+L_{\tilde{I}_{k}}\right)\right]<1 .
$$

Proof In the sequel, the notation $B_{r}(x, Z)$ stands for the closed ball with center at $x$ and radius $r>0$ in $Z$, where $\left(Z,\|\cdot\|_{Z}\right)$ is a Banach space. Let $y:(-\infty, T] \rightarrow H$ be defined by

$$
y(t)= \begin{cases}\phi(t), & t \in(-\infty, 0] \\ C(t) \phi(0)+S(t) \psi, & t \in J\end{cases}
$$

On the space $Y=\{x \in \mathcal{P C}: x(0)=\phi(0)\}$ endowed with the uniform convergence topology, we define the operator $\Phi: Y \rightarrow Y$ by

$$
\begin{aligned}
\Phi x(t)= & C(t) \phi(0)+S(t)[\psi-g(0, \phi)]+\int_{0}^{t} C(t-s) g\left(s, \bar{x}_{s}\right) \mathrm{d} s \\
& +\int_{0}^{t} S(t-s) f\left(s, \bar{x}_{s}\right) \mathrm{d} s+\int_{0}^{t} S(t-s) \sigma\left(s, \bar{x}_{s}\right) \mathrm{d} w(s) \\
& +\sum_{0<t_{k}<t} C\left(t-t_{k}\right) I_{k}\left(\bar{x}_{t_{k}}\right)+\sum_{0<t_{k}<t} S\left(t-t_{k}\right) \tilde{I}_{k}\left(\bar{x}_{t_{k}}\right), \quad t \in J,
\end{aligned}
$$


where $\bar{x}$ is such that $\bar{x}_{0}=\phi$ and $\bar{x}=x$ on $J$. From Lemma 2 and the assumption on $\phi$, we infer that $\Phi x \in \mathcal{P C}$. Our proof will be split into the following three steps.

Step 1. In what follows, we prove that there exists $r>0$ such that $\Phi\left(B_{r}\left(\left.y\right|_{J}, Y\right)\right) \subseteq$ $B_{r}\left(\left.y\right|_{J}, Y\right)$. In fact, if it is not true, then for each $r>0$ there exist $x^{r} \in B_{r}\left(\left.y\right|_{J}, Y\right)$ and $t^{r} \in J$ such that $r<E\left\|\Phi\left(x^{r}\left(t^{r}\right)\right)-y\left(t^{r}\right)\right\|^{2}$. Therefore, from Lemma 2 and the assumptions, we have

$$
\begin{aligned}
& r^{2}<E\left\|\Phi\left(x^{r}\left(t^{r}\right)\right)-y\left(t^{r}\right)\right\|^{2} \\
& \leq 6 E\left\|S\left(t^{r}\right) g(0, \phi)\right\|^{2}+6 E\left(\int_{0}^{t^{r}} C\left(t^{r}-s\right) g\left(s, \bar{x}_{s}^{r}\right) \mathrm{d} s\right)^{2} \\
& +6 E\left(\int_{0}^{t^{r}} S\left(t^{r}-s\right) f\left(s, \bar{x}_{s}^{r}\right) \mathrm{d} s\right)^{2}+6 M \operatorname{Tr}(Q) E \int_{0}^{t^{r}}\left\|\sigma\left(s, \bar{x}_{s}^{r}\right)\right\|^{2} \mathrm{~d} s \\
& +6 E\left(\sum_{0<t_{k}<t} C\left(t-t_{k}\right) I_{k}\left(\bar{x}_{t_{k}}^{r}\right)\right)^{2}+6 E\left(\sum_{0<t_{k}<t} S\left(t-t_{k}\right) \tilde{I}_{k}\left(\bar{x}_{t_{k}}^{r}\right)\right)^{2} \\
& \leq 12 M L_{g}\left(\|\phi\|_{\mathcal{B}_{h}}^{2}+1\right)+6 T M E \int_{0}^{t^{r}}\left\|g\left(s, \bar{x}_{s}^{r}\right)\right\|^{2} \mathrm{~d} s+6 T M E \int_{0}^{t^{r}}\left\|f\left(s, \bar{x}_{s}^{r}\right)\right\|^{2} \mathrm{~d} s \\
& +6 M \operatorname{Tr}(Q) E \int_{0}^{t^{r}}\left\|\sigma\left(s, \bar{x}_{s}^{r}\right)\right\|^{2} \mathrm{~d} s+6 M \sum_{0<t_{k}<t} E\left(I_{k}\left(\bar{x}_{t_{k}}^{r}\right)\right)^{2}+6 M \sum_{0<t_{k}<t} E\left(\tilde{I}_{k}\left(\bar{x}_{t_{k}}^{r}\right)\right)^{2} \\
& \leq 12 M L_{g}\left(\|\phi\|_{\mathcal{B}_{h}}^{2}+1\right)+12 T M E \int_{0}^{t^{r}}\left\|g\left(s, \bar{x}_{s}^{r}\right)-g\left(s, y_{s}\right)\right\|^{2} \mathrm{~d} s \\
& +12 T M E \int_{0}^{t^{r}}\left\|g\left(s, y_{s}\right)\right\|^{2} \mathrm{~d} s+6 T M E \int_{0}^{t^{r}}\left\|f\left(s, \bar{x}_{s}^{r}\right)\right\|^{2} \mathrm{~d} s \\
& +12 M \operatorname{Tr}(Q) E \int_{0}^{t^{r}}\left\|\sigma\left(s, \bar{x}_{s}^{r}\right)-\sigma\left(s, y_{s}\right)\right\|^{2} \mathrm{~d} s+12 M \operatorname{Tr}(Q) E \int_{0}^{t^{r}}\left\|\sigma\left(s, y_{s}\right)\right\|^{2} \mathrm{~d} s \\
& +12 M \sum_{0<t_{k}<t} E\left[\left\|I_{k}\left(\bar{x}_{t_{k}}^{r}\right)-I_{k}\left(y_{t_{k}}\right)\right\|^{2}+\left\|I_{k}\left(y_{t_{k}}\right)\right\|^{2}\right] \\
& +12 M \sum_{0<t_{k}<t} E\left[\left\|\tilde{I}_{k}\left(\bar{x}_{t_{k}}^{r}\right)-\tilde{I}_{k}\left(y_{t_{k}}\right)\right\|^{2}+\left\|\tilde{I}_{k}\left(y_{t_{k}}\right)\right\|^{2}\right] \\
& \leq 12 M L_{g}\left(\|\phi\|_{\mathcal{B}_{h}}^{2}+1\right)+24 l^{2} M\left(T L_{g}+\operatorname{Tr}(Q) L_{\sigma}\right) \int_{0}^{t^{r}} \sup _{0 \leq u \leq s} E\left\|\bar{x}^{r}(u)-y(u)\right\|^{2} \mathrm{~d} s \\
& +24 M\left(T L_{g}+\operatorname{Tr}(Q) L_{\sigma}\right) \int_{0}^{t^{r}}\left(\left\|y_{s}\right\|_{\mathcal{B}_{h}}^{2}+1\right) \mathrm{d} s \\
& +6 T M E \int_{0}^{t^{r}} m(s) \Psi\left(2\|\phi\|_{\mathcal{B}_{h}}^{2}+2 l^{2}\left(r^{2}+\|y\|_{T}^{2}\right)\right) \mathrm{d} s \\
& +24 l^{2} M r^{2} \sum_{k=1}^{m}\left[L_{I_{k}}+L_{\tilde{I}_{k}}\right]+12 M \sum_{k=1}^{m}\left[E\left\|I_{k}\left(y_{t_{k}}\right)\right\|^{2}+E\left\|\tilde{I}_{k}\left(y_{t_{k}}\right)\right\|^{2}\right],
\end{aligned}
$$

where $\|y\|_{T}=\sup _{0 \leq s \leq T} E\|y(s)\|$. Dividing both sides by $r^{2}$ and taking the limit as $r \rightarrow \infty$, we obtain

$$
1 \leq 12 M l^{2}\left[T \Lambda \int_{0}^{T} m(s) \mathrm{d} s+2 \sum_{k=1}^{m}\left(L_{I_{k}}+L_{\tilde{I}_{k}}\right)+2 T\left(T L_{g}+\operatorname{Tr}(Q) L_{\sigma}\right)\right]
$$


which contradicts (6). Thus, for some positive number $r, \Phi\left(B_{r}\left(\left.y\right|_{J}, Y\right)\right) \subseteq B_{r}\left(\left.y\right|_{J}, Y\right)$. In what follows, we aim to show that the operator $\Phi$ has a fixed point on $B_{r}\left(\left.y\right|_{J}, Y\right)$, which implies that (1)-(4) has a mild solution. To this end, we decompose $\Phi$ as $\Phi=\Phi_{1}+\Phi_{2}$, where $\Phi_{1}$, $\Phi_{2}$ are defined on $B_{r}\left(\left.y\right|_{J}, Y\right)$, respectively, by

$$
\begin{aligned}
\left(\Phi_{1} x\right)(t)= & C(t) \phi(0)+S(t)[\psi-g(0, \phi)] \\
& +\int_{0}^{t} C(t-s) g\left(s, \bar{x}_{s}\right) \mathrm{d} s+\int_{0}^{t} S(t-s) \sigma\left(s, \bar{x}_{s}\right) \mathrm{d} w(s) \\
& +\sum_{0<t_{k}<t} C\left(t-t_{k}\right) I_{k}\left(\bar{x}_{t_{k}}\right)+\sum_{0<t_{k}<t} S\left(t-t_{k}\right) \tilde{I}_{k}\left(\bar{x}_{t_{k}}\right)
\end{aligned}
$$

and

$$
\left(\Phi_{2} x\right)(t)=\int_{0}^{t} S(t-s) f\left(s, \bar{x}_{s}\right) \mathrm{d} s
$$

for $t \in J$. We will show that $\Phi_{1}$ is a contraction and $\Phi_{2}$ is completely continuous.

Step 2. $\Phi_{1}$ is a contraction. Let $x, y \in B_{r}\left(\left.y\right|_{J}, Y\right)$. Then, for each $t \in J$, we have

$$
\begin{aligned}
& E\left\|\left(\Phi_{1} x\right)(t)-\left(\Phi_{1} y\right)(t)\right\|^{2} \\
& \leq 4 E\left\|\int_{0}^{t} C(t-s)\left[g\left(s, \bar{x}_{s}\right)-g\left(s, \bar{y}_{s}\right)\right] \mathrm{d} s\right\|^{2} \\
& \quad+4 E\left\|\int_{0}^{t} S(t-s)\left[\sigma\left(s, \bar{x}_{s}\right)-\sigma\left(s, \bar{y}_{s}\right)\right] \mathrm{d} w(s)\right\|^{2} \\
& \quad+4 E\left\|\sum_{0<t_{k}<t} C\left(t-t_{k}\right)\left(I_{k}\left(\bar{x}_{t_{k}}\right)-I_{k}\left(\bar{y}_{t_{k}}\right)\right)\right\|^{2} \\
& \quad+4 E\left\|\sum_{0<t_{k}<t} S\left(t-t_{k}\right)\left(\tilde{I}_{k}\left(\bar{x}_{t_{k}}\right)-\tilde{I}_{k}\left(\bar{y}_{t_{k}}\right)\right)\right\|^{2} \\
& \leq 4 M\left(T L_{g}+\operatorname{Tr}(Q) L_{\sigma}\right) \int_{0}^{t}\left\|\bar{x}_{s}-\bar{y}_{s}\right\|_{\mathcal{B}_{h}}^{2} \mathrm{~d} s+4 M \sum_{k=1}^{m}\left(L_{I_{k}}+L_{\tilde{I}_{k}}\right)\left\|\bar{x}_{t_{k}}-\bar{y}_{t_{k}}\right\|_{\mathcal{B}_{h}}^{2} \\
& \leq 8 M l^{2}\left(T L_{g}+\operatorname{Tr}(Q) L_{\sigma}\right) \int_{0}^{t} \sup _{0 \leq u \leq s} E\|\bar{x}(u)-\bar{y}(u)\|_{\mathcal{B}_{h}}^{2} \mathrm{~d} s \\
& \quad+8 M l^{2} \sum_{k=1}^{m} \sup _{0 \leq s \leq T} E\|\bar{x}(s)-\bar{y}(s)\|^{2}\left(L_{I_{k}}+L_{\tilde{I}_{k}}\right) .
\end{aligned}
$$

Therefore, we get

$$
\left\|\left(\Phi_{1} x\right)(t)-\left(\Phi_{1} y\right)(t)\right\|_{\mathcal{P C}}^{2} \leq L_{0}\|\bar{x}-\bar{y}\|_{\mathcal{P C}}^{2}
$$

where $L_{0}=8 M l^{2}\left[T\left(T L_{g}+\operatorname{Tr}(Q) L_{\sigma}\right)+\sum_{k=1}^{m}\left(L_{I_{k}}+L_{\tilde{I}_{k}}\right)\right]$. Thus, we obtain

$$
\left\|\Phi_{1} x-\Phi_{1} y\right\|_{\mathcal{P C}}^{2} \leq L_{0}\|x-y\|_{\mathcal{P C}}^{2} .
$$

By (7), we see that $\Phi_{1}$ is a contraction on $B_{r}\left(\left.y\right|_{J}, Y\right)$. 
Step 3. $\Phi_{2}$ is completely continuous on $B_{r}\left(\left.y\right|_{J}, Y\right)$.

Claim $1 \Phi_{2}$ maps bounded sets to bounded sets in $B_{r}\left(\left.y\right|_{J}, Y\right)$.

In the sequel, $r^{*}, r^{* *}$ are the numbers defined by $r^{*}:=2 l^{2} \sup _{0 \leq s \leq t}\left(E\|x(s)\|^{2}\right)+2\|\phi\|_{\mathcal{B}_{h}}^{2}$ and $r^{* *}:=M \Psi\left(r^{*}\right) \int_{0}^{t} m(s) \mathrm{d} s$, respectively.

$$
\begin{aligned}
E\left\|\left(\Phi_{2} x\right)(t)\right\|^{2} & \leq \int_{0}^{t}\|s(t-s)\|^{2} E\left\|f\left(s, \bar{x}_{s}\right)\right\|^{2} \mathrm{~d} s \\
& \leq M \int_{0}^{t} m(t) \Psi\left(\left\|\bar{x}_{s}\right\|_{\mathcal{B}_{h}}^{2}\right) \mathrm{d} s \\
& \leq M \int_{0}^{t} m(t) \Psi\left(2 l^{2} \sup _{0 \leq s \leq t}\left(E\|x(s)\|^{2}\right)+2\|\phi\|_{\mathcal{B}_{h}}^{2}\right) \mathrm{d} s \\
& \leq M \Psi\left(r^{*}\right) \int_{0}^{t} m(s) \mathrm{d} s \\
& =r^{* *},
\end{aligned}
$$

which shows the desired result of the claim.

Claim 2 The set of functions $\Phi_{2}\left(B_{r}\left(\left.y\right|_{J}, Y\right)\right)$ is equicontinuous on $J$.

Let $\varepsilon>0$ small enough and $0<t_{1}<t_{2}$. We get

$$
\begin{aligned}
E\left\|\left(\Phi_{2} x\right)\left(t_{2}\right)-\left(\Phi_{2} x\right)\left(t_{1}\right)\right\|^{2} \leq & 3 T \int_{0}^{t_{1}-\varepsilon}\left\|S\left(t_{2}-s\right)-S\left(t_{1}-s\right)\right\|^{2} E\left\|f\left(s, \bar{x}_{s}\right)\right\|^{2} \mathrm{~d} s \\
& +3 \varepsilon \int_{t_{1}-\varepsilon}^{t_{1}}\left\|S\left(t_{2}-s\right)-S\left(t_{1}-s\right)\right\|^{2} E\left\|f\left(s, \bar{x}_{s}\right)\right\|^{2} \mathrm{~d} s \\
& +3\left(t_{2}-t_{1}\right) \int_{t_{1}}^{t_{2}}\left\|S\left(t_{2}-s\right)\right\|^{2} E\left\|f\left(s, \bar{x}_{s}\right)\right\|^{2} \mathrm{~d} s \\
\leq & 3 T \Psi\left(r^{*}\right) \int_{0}^{t_{1}-\varepsilon}\left\|S\left(t_{2}-s\right)-S\left(t_{1}-s\right)\right\|^{2} m(s) \mathrm{d} s \\
& +3 \varepsilon \Psi\left(r^{*}\right) \int_{t_{1}-\varepsilon}^{t_{1}}\left\|S\left(t_{2}-s\right)-S\left(t_{1}-s\right)\right\|^{2} m(s) \mathrm{d} s \\
& +3\left(t_{2}-t_{1}\right) \Psi\left(r^{*}\right) \int_{t_{1}}^{t_{2}}\left\|S\left(t_{2}-s\right)\right\|^{2} m(s) \mathrm{d} s,
\end{aligned}
$$

which proves that $\Phi_{2}\left(B_{r}\left(\left.y\right|_{J}, Y\right)\right)$ is equicontinuous on $J$.

Claim $3 \Phi_{2}$ maps $\left(B_{r}\left(\left.y\right|_{J}, Y\right)\right)$ into a precompact set in $\left(B_{r}\left(\left.y\right|_{J}, Y\right)\right)$. That is, for each fixed $t \in J$, the set $V(t)=\left\{\Phi_{2} z(t): z \in\left(B_{r}\left(\left.y\right|_{J}, Y\right)\right)\right\}$ is precompact in $\left(B_{r}\left(\left.y\right|_{J}, Y\right)\right)$.

Obviously, $V(0)=\left\{\Phi_{2}(0)\right\}$. Let $t>0$ fixed and for $0<\varepsilon<t$, define

$$
\left(\Phi_{2}^{\varepsilon} x\right)(t)=S(\varepsilon) \int_{0}^{t-\varepsilon} S(t-\varepsilon-s) f\left(s, \bar{x}_{s}\right) \mathrm{d} s
$$


Since $S(t)$ is a compact operator, the set $V^{\varepsilon}(t)=\left\{\Phi_{2}^{\varepsilon} x(t): x \in\left(B_{r}\left(\left.y\right|_{J}, Y\right)\right)\right\}$ is relatively compact in $H$ for every $\varepsilon, 0<\varepsilon<t$. Moreover, for each $x \in\left(B_{r}\left(\left.y\right|_{J}, Y\right)\right)$, we have

$$
\begin{aligned}
E\left\|\left(\Phi_{2} x\right)(t)-\left(\Phi_{2}^{\varepsilon} x\right)(t)\right\|^{2} & \leq \varepsilon \int_{t-\varepsilon}^{t}\|S(t-s)\|^{2} E\left\|f\left(s, \bar{x}_{s}\right)\right\|^{2} \mathrm{~d} s \\
& \leq M \varepsilon \int_{t-\varepsilon}^{t} m(t) \Psi\left(\left\|\bar{x}_{s}\right\|_{\mathcal{B}_{h}}^{2}\right) \mathrm{d} s \\
& \leq M \Psi\left(r^{*}\right) \varepsilon \int_{t-\varepsilon}^{t} m(s) \mathrm{d} s .
\end{aligned}
$$

Therefore, we have

$$
E\left\|\left(\Phi_{2} x\right)(t)-\left(\Phi_{2}^{\varepsilon} x\right)(t)\right\|^{2} \rightarrow 0, \quad \text { as } \varepsilon \rightarrow 0^{+},
$$

and there are precompact sets arbitrary close to the set $V(t)=\left\{\Phi_{2} x(t): x \in\left(B_{r}\left(\left.y\right|_{J}, Y\right)\right)\right\}$. Thus, the set $V(t)=\left\{\Phi_{2} x(t): x \in\left(B_{r}\left(\left.y\right|_{J}, Y\right)\right)\right\}$ is precompact in $\left(B_{r}\left(\left.y\right|_{J}, Y\right)\right)$. Therefore, from the Arzela-Ascoli theorem, the operator $\Phi_{2}$ is completely continuous. From Theorem 5, we infer that there exists a mild solution for the system (1)-(4).

\section{Examples}

In this section, two types of stochastic nonlinear wave equations with infinite delay and impulsive effects are provided to illustrate the theory obtained.

Example 8 We consider the following second-order stochastic Volterra integro-differential equations with initial-boundary conditions and impulsive effects:

$$
\begin{aligned}
& \mathrm{d}\left[\frac{\partial x(t, \xi)}{\partial t}-\int_{-\infty}^{t} K_{1}(t, s) F_{1}(x(s, \xi)) \mathrm{d} s\right] \\
& =\frac{\partial^{2} x(t, \xi)}{\partial \xi^{2}} \mathrm{~d} t+\left(\int_{-\infty}^{t} K_{2}(t, s) F_{2}(x(s, \xi)) \mathrm{d} s\right) \mathrm{d} t \\
& \quad+\left(\int_{-\infty}^{t} K_{3}(t, s) F_{3}(x(s, \xi)) \mathrm{d} s\right) \mathrm{d} w(t), \\
& \quad 0<\xi<\pi, 0 \leq t \leq T, t \neq t_{k}, \\
& \quad k=1,2, \ldots, m, \\
& x(t, \xi)=\phi(t, \xi), \quad-\infty<t \leq 0,0<\xi<\pi, \\
& x(t, 0)=x(t, \pi)=0, \quad 0 \leq t \leq T, \\
& \frac{\partial x(0, \xi)}{\partial t}=x_{1}(\xi), \quad 0<\xi<\pi, \\
& x\left(t_{k}^{+}\right)-x\left(t_{k}^{-}\right)=I_{k}\left(x\left(t_{k}\right)\right), \quad x^{\prime}\left(t_{k}^{+}\right)-x^{\prime}\left(t_{k}^{-}\right)=\tilde{I}_{k}\left(x\left(t_{k}\right)\right), \\
& k=1,2, \ldots, m, \quad
\end{aligned}
$$

where $w(t)$ is a standard cylindrical Wiener process in $\mathbb{R}$ defined on the probability space $(\Omega, \mathcal{F}, P)$. 
Let $H=L_{2}[0, \pi]$. The operator $A$ is defined by

$$
(A z)(\xi)=\frac{\mathrm{d}^{2} z(\xi)}{\mathrm{d} \xi^{2}}, \quad \text { with domain } D(A)=\{z \in H: z(0)=z(\pi)\} .
$$

The spectrum of $A$ consists of the eigenvalues $-n^{2}$ for $n \in \mathbb{N}$, with associated eigenvectors $z_{n}(\xi)=\left(\frac{2}{\pi}\right)^{1 / 2} \sin (n \xi)$. Furthermore, the set $\left\{z_{n} ; n \in \mathbb{N}\right\}$ is an orthonormal basis of $H$. In particular,

$$
A x=\sum_{n=1}^{\infty}-n^{2}\left\langle x, z_{n}\right\rangle z_{n}, \quad x \in D(A) .
$$

The operators $C(t)$ defined by

$$
C(t) x=\sum_{n=1}^{\infty} \cos (n t)\left\langle x, z_{n}\right\rangle z_{n}, \quad t \in \mathbb{R},
$$

form a cosine function on $H$, with associated sine function

$$
S(t) x=\sum_{n=1}^{\infty} \frac{\sin (n t)}{n}\left\langle x, z_{n}\right\rangle z_{n}, \quad t \in \mathbb{R} .
$$

From [17], for all $x \in H, t \in \mathbb{R},\|S(t)\| \leq 1$ and $\|C(t)\| \leq 1$.

Let $K_{i}(t, s) \in C\left(\mathbb{R}^{2}, \mathbb{R}\right), i=1,2,3$ and assume that there exists a positive continuous function $f(s)$ on $\mathbb{R}_{-}$such that

$$
\left|K_{i}(t, t+s)\right| \leq f(s), \quad i=1,2,3, \quad l_{0}=\int_{-\infty}^{0} f(s) \mathrm{d} s<\infty .
$$

Now, we give the phase space $\mathcal{B}_{h}$. Assume that $h:(-\infty, 0] \rightarrow(0, \infty)$ is a continuous function with $l=\int_{-\infty}^{0} h(t) \mathrm{d} t<\infty$. For any $a>0$, define

$$
\begin{gathered}
\mathcal{B}_{h}=\left\{\psi:(-\infty, 0] \rightarrow H:\left(E|\psi(\theta)|^{2}\right)^{1 / 2}\right. \text { is a bounded and measurable } \\
\text { function on } \left.[-a, 0] \text { and } \int_{-\infty}^{0} h(s)\left(E|\psi(s)|^{2}\right)^{1 / 2} \mathrm{~d} s<\infty\right\}
\end{gathered}
$$

We endow $\mathcal{B}_{h}$ with the norm

$$
\|\psi\|_{\mathcal{B}_{h}}=\int_{-\infty}^{0} h(s)\left(E|\psi(s)|^{2}\right)^{1 / 2} \mathrm{~d} s, \quad \text { for all } \psi \in \mathcal{B}_{h} .
$$

Then $\left(\mathcal{B}_{h},\|\cdot\|_{\mathcal{B}_{h}}\right)$ is a Banach space. Let

$$
\begin{aligned}
& \phi(\xi)=\int_{-\infty}^{0} h(s) \phi(s, \xi) \mathrm{d} s, \quad g(t, \phi)(\xi)=\int_{-\infty}^{0} K_{1}(t, t+s) F_{1}(\phi(s, \xi)) \mathrm{d} s \\
& f(t, \phi)(\xi)=\int_{-\infty}^{0} K_{2}(t, t+s) F_{2}(\phi(s, \xi)) \mathrm{d} s, \quad \sigma(t, \phi)(\xi)=\int_{-\infty}^{0} K_{3}(t, t+s)(\phi(s, \xi)) \mathrm{d} s
\end{aligned}
$$


Then (8)-(12) can be rewritten in the abstract form (1)-(4). We can propose suitable conditions on the coefficients appeared in the above equation to guarantee (8)-(12) has at least one mild solution by means of Theorem 7 .

Example 9 We consider the following stochastic nonlinear wave equation with impulsive effects and infinite delay:

$$
\begin{aligned}
& \mathrm{d}\left[\frac{\partial x(t, \xi)}{\partial t}-f_{1}(t, x(t-r, \xi))\right] \\
& =\frac{\partial^{2} x(t, \xi)}{\partial \xi^{2}} \mathrm{~d} t+f_{2}(t, x(t-r, \xi)) \mathrm{d} t \\
& \quad+\sigma(t, x(t-r, \xi)) \mathrm{d} w(t), \\
& \quad 0 \leq \xi \leq \pi, 0 \leq t \leq T, r>0, t \neq t_{k}, k=1,2, \ldots, m, \\
& x(t, \xi)=\phi(t, \xi), \quad-\infty<t \leq 0,0<\xi<\pi, \\
& x(t, 0)=x(t, \pi)=0, \quad 0 \leq t \leq T, \\
& \frac{\partial x(0, \xi)}{\partial t}=x_{1}(\xi), \quad 0<\xi<\pi, \\
& x\left(t_{k}^{+}\right)-x\left(t_{k}^{-}\right)=I_{k}\left(x\left(t_{k}\right)\right), \quad x^{\prime}\left(t_{k}^{+}\right)-x^{\prime}\left(t_{k}^{-}\right)=\tilde{I}_{k}\left(x\left(t_{k}\right)\right), \\
& k=1,2, \ldots, m,
\end{aligned}
$$

where $x_{1} \in L_{0}^{2}(\Omega ; H), \phi \in \mathcal{B}_{h}, \mathcal{B}_{h}$ is defined as Example $8, H=L^{2}([0, \pi])$, and $w$ is an $H$-valued Wiener process.

Let $A, C(t)$ and $S(t)$ be defined as Example 8. Then the above system (13)-(17) can be rewritten in the form of (1)-(4). Further, we assume that $f_{i}:[0, T] \times \mathbb{R} \rightarrow \mathbb{R}(i=1,2)$, $\sigma:[0, T] \times \mathbb{R} \rightarrow B L(H)$ and $I_{k}, \tilde{I}_{k}$ satisfy (H2)-(H5). Then (13)-(17) has at least one mild solution.

\section{Competing interests}

The author declares that he has no competing interests.

\section{Author's contributions}

The author is the only person who is responsible to this work.

\section{Acknowledgements}

The author sincerely thanks the reviewers for their valuable suggestions and useful comments. This work is partially supported by Mathematical discipline project of Anhui Agricultural University (Project number. XKXWD2013020; XK2013029).

Received: 23 October 2013 Accepted: 14 April 2014 Published: 30 Apr 2014

\section{References}

1. Hino, Y, Murakami, S, Naito, T: Functional Differential Equations with Infinite Delay. Lecture Notes in Mathematics, vol. 1473. Springer, Berlin (1991)

2. Caraballo, T: Asymptotic exponential stability of stochastic partial differential equations with delay. Stochastics 33 , 27-47 (1990)

3. Da Prato, G, Zabczyk, J: Stochastic Equations in Infinite Dimensions. Cambridge University Press, Cambridge (1992)

4. Mahmudov, NI: Existence and uniqueness results for neutral SDEs in Hilbert spaces. Stoch. Anal. Appl. 24, 79-95 (2006)

5. Mao, X: Stochastic Differential Equations and Applications. Horwood, Chichester (1997)

6. Hale, JK, Lunel, SMV: Introduction to Functional Differential Equations. Springer, Berlin (1991)

7. Kolmanovskii, VB, Myshkis, A: Applied Theory of Functional Differential Equations. Kluwer Academic, Norwell (1992) 
8. Govindan, TE: Exponential stability in mean-square of parabolic quasilinear stochastic delay evolution equations. Stoch. Anal. Appl. 17, 443-461 (1999)

9. Ren, Y, Chen, L: A note on the neutral stochastic functional differential equations with infinite delay and Poisson jumps in an abstract space. J. Math. Phys. 50, 082704 (2009)

10. Ren, $Y, L u, S, X i a, N$ : Remarks on the existence and uniqueness of the solutions to stochastic functional differential equations with infinite delay. J. Comput. Appl. Math. 220, 364-372 (2008)

11. Ren, Y, Xia, N: Existence, uniqueness and stability of the solutions to neutral stochastic functional differential equations with infinite delay. Appl. Math. Comput. 210, 72-79 (2009)

12. Ren, $Y, X i a, N$ : A note on the existence and uniqueness of the solution to neutral stochastic functional differential equations with infinite delay. Appl. Math. Comput. 214, 457-461 (2009)

13. Nieto, JJ, Rodriguez-Lopez, R: New comparison results for impulsive integro-differential equations and applications. J. Math. Anal. Appl. 328, 1343-1368 (2007)

14. Samoilenko, AM, Perestyuk, NA: Impulsive Differential Equations. World Scientific, Singapore (1995)

15. Travis, CC, Webb, GF: Cosine families and abstract nonlinear second order differential equations. Acta Math. Acad. Sci. Hung. 32, 76-96 (1978)

16. Fattorini, HO: Second Order Linear Differential Equations in Banach Spaces. North-Holland Mathematics Studies, vol. 108. North-Holland, Amsterdam (1985)

17. Travis, CC, Webb, GF: Second order differential equations in Banach space. In: Proceedings of an International Symposium on Nonlinear Equations in Abstract Spaces, pp. 331-361. Academic Press, New York (1987)

18. McKibben, MA: Second-order damped functional stochastic evolution equations in Hilbert space. Dyn. Syst. Appl. 12, 467-487 (2003)

19. Mahmudov, NI, McKibben, MA: Abstract second-order damped McKean-Vlasov stochastic evolution equations. Stoch. Anal. Appl. 24, 303-328 (2006)

20. McKibben, MA: Second-order neutral stochastic evolution equations with heredity. J. Appl. Math. Stoch. Anal. 2004, 177-192 (2004)

21. Balasubramaniam, P, Muthukumar, P: Approximate controllability of second-order stochastic distributed implicit functional differential systems with infinite delay. J. Optim. Theory Appl. 143, 225-244 (2009)

22. Da Prato, G: Non-linear stochastic partial differential equations. In: Mathematics of Complexity and Dynamical Systems, pp. 1126-1136. Springer, Berlin (2011)

23. Li, Y, Liu, B: Existence of solution of nonlinear neutral functional differential inclusions with infinite delay. Stoch. Anal. Appl. 25, 397-415 (2007)

24. Burton, TA, Kirk, C: A fixed point theorem of Krasnoselskii-Schaefer type. Math. Nachr. 189, $23-31$ (1998)

10.1186/1687-1847-2014-112

Cite this article as: Yue: Second-order neutral impulsive stochastic evolution equations with infinite delay. Advances in Difference Equations 2014, 2014:112

\section{Submit your manuscript to a SpringerOpen ${ }^{\circ}$ journal and benefit from:}

- Convenient online submission

- Rigorous peer review

- Immediate publication on acceptance

- Open access: articles freely available online

- High visibility within the field

- Retaining the copyright to your article 